\title{
Mid-IR Spectroscopy of Submm Galaxies: Extended Star Formation in High- $z$ Galaxies
}

\author{
K. Menéndez-Delmestre ${ }^{1}$, A. W. Blain ${ }^{2}$, I. Smail ${ }^{3}$, D. M. Alexander ${ }^{4}$,

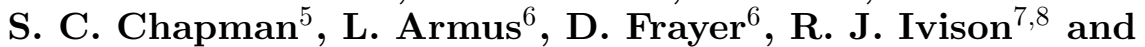 \\ H. Teplitz ${ }^{6}$ \\ ${ }^{1}$ NSF Astronomy \& Astrophysics Postdoctoral Fellow; Carnegie Observatories, Pasadena, CA \\ email: kmd@obs.carnegiescience.edu ${ }^{2}$ Caltech, Pasadena, CA \\ ${ }^{3}$ Computational Cosmology, Durham, UK ${ }^{4}$ Physics, Durham, UK \\ ${ }^{5}$ Inst. of Astronomy, Cambridge, UK ${ }^{6}$ SSC, Pasadena, CA ${ }^{7}$ Astronomy Technology Centre, \\ Edinburgh, UK ${ }^{8}$ Inst. for Astronomy, Edinburgh, UK
}

\begin{abstract}
Ultra-luminous infrared galaxies (ULIRGs; L $>10^{12} \mathrm{~L}_{\odot}$ ) are quite rare in the local universe, but seem to dominate the co-moving energy density at $z>2$. Many are opticallyfaint, dust-obscured galaxies that have been identified only relatively recently by the detection of their thermal dust emission redshifted into the sub-mm wavelengths. These submm galaxies (SMGs) have been shown to be a massive objects $\left(\mathrm{M}_{\star} \sim 10^{11} \mathrm{M}_{\odot}\right)$ undergoing intense starformation(SFRs $\sim 10^{2}-10^{3} \mathrm{M}_{\odot} \mathrm{yr}^{-1}$ ) and the likely progenitors of massive ellipticals today. However, the AGN contribution to the far-IR luminosity had for years remained a caveat to these results. We used the Spitzer Infrared Spectrograph (IRS) to investigate the energetics of 24 radio-identified and spectroscopically-confirmed SMGs in the redshift range of $0.6<z<3.2$. We find emission from Polycyclic Aromatic Hydrocarbons (PAHs) - which are associated with intense star-formation activity - in $>80 \%$ of our sample and find that the median mid-IR spectrum is well described by a starburst component with an additional power-law continuum representing $<32 \%$ AGN contribution to the far-IR luminosity. We also find evidence for a more extended distribution of warm dust in SMGs compared to the more compact nuclear bursts in local ULIRGs and starbursts, suggesting that SMGs are not simple high-redshift analogs of local ULIRGs or nuclear starbursts, but have star formation which resembles that seen in less-extreme star-forming environments at $z \sim 0$.
\end{abstract}

Keywords. infrared: galaxies — galaxies: starburst — galaxies: AGN — galaxies: submillimeter

Deep X-ray studies suggest that $>28-50 \%$ of SMGs host an obscured AGN (Alexander et al. 2005). Although X-rays provide one of the most direct routes to estimate the luminosities of AGN, under high column densities such as found in SMGs, hard X-ray photons may be completely absorbed. The mid-IR provides an indirect insight to the dust-enshrouded nature of SMGs. We present the largest sample of SMGs observed in the mid-IR with Spitzer IRS (see Menéndez-Delmestre et al. 2009, hereafter MD09, for full discussion). Our main results are:

1. We find that $>80 \%$ of the SMGs in our sample display luminous PAH features and only four SMGs have continuum-dominated spectra (weak or absent PAH features). This indicates that, although some diversity exists within the population, SMGs are in general a population dominated by intense star-forming activity.

2. The composite SMG spectrum is best fit by the combination of a starburst template and an additional power-law continuum representing a maximum AGN contribution of $<32 \%$ to the far-IR luminosity in SMGs (see Fig. 1).

3 . We quantify the strength of the silicate absorption feature and find that the distribution in $\tau_{9.7 \mu \mathrm{m}}$ for SMGs falls below that of local ULIRGs and the most obscured low-redshift nuclear starburst-dominated galaxies. This suggests that SMGs have lower dust obscuration to their mid-IR continuum emitting regions than these local samples.

4. Comparison of 7.7/11.3 PAH flux ratios suggests that SMGs host similar radiation envi- 


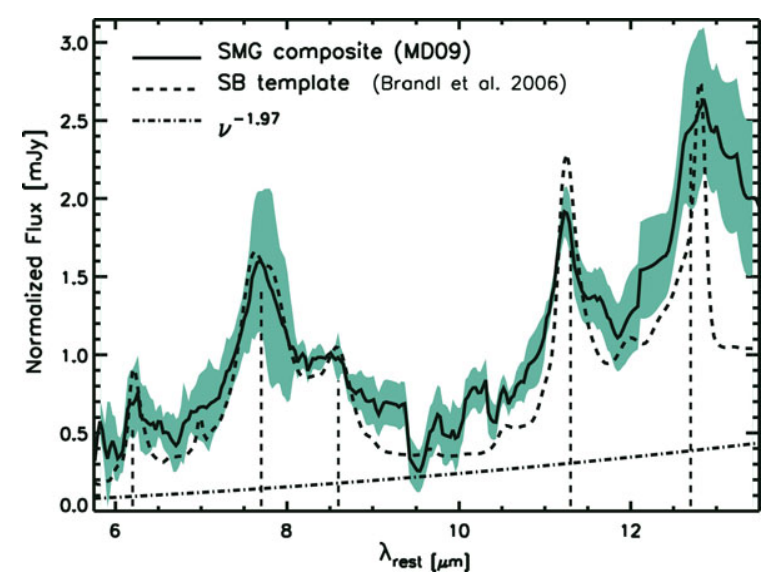

Figure 1. The SMG composite spectrum (solid line) is dominated by strong PAH emission features (vertical dashed lines). We fit this composite with a local starburst template (dashed spectrum) and derive an upper limit to the contribution from an AGN by making the conservative assumption that the additional continuum emission (dot-dashed line) arises solely from an obscured AGN. As described in MD09, we estimate that AGN activity contributes $<32 \%$ to the far-IR luminosity in typical SMGs. We emphasize that this is a strong upper limit for the SMG population, since a fraction of the red continuum in this composite spectrum likely arises from dust emission heated by optically-thick HII regions.

ronments to local starbursts. However, the 7.7/6.2 PAH ratio is lower in SMGs than in local nuclear starbursts. A stronger 6.2- $\mu \mathrm{m}$ PAH emission relative to the $7.7 \mu \mathrm{m}$ PAH feature may be attributed to lower extinction by ice (at $6 \mu \mathrm{m}$ ) along the line of sight to SMGs

We conclude that the detailed mid-IR spectral properties of SMGs show several differences to local ULIRGs and nuclear starbursts. The differences in Si-absorption strengths and 7.7/6.2 PAH ratios can be most easily explained by a difference in the extinction to the mid-IR continuum and line emitting regions of these galaxies, with the SMGs showing systematically lower extinction. Considering that SMGs are very dusty objects, a lower extinction suggests that the warm dust is distributed over spatial scales significantly more extended in SMGs than found in local ULIRGs and nuclear starbursts.

\section{References}

Alexander, D. M., Bauer, F. E., Chapman, S., Smail, I., Blain, A., Brandt, W. N., \& Ivison, R. 2005, ApJ, 632, 736

Brandl, B. R. et al. 2006, ApJ, 653, 1129

Chapman, S., Blain, A., Smail, I., \& Ivison, R. 2005, ApJ, 622, 772

Krabbe, A., Böker, T., \& Maiolino, R. 2001, ApJ, 557, 626

Menéndez-Delmestre, K. et al. 2009, ApJ, 699, 667 (MD09) 